\title{
MiR-200c sensitizes clear-cell renal cell carcinoma cells to sorafenib and imatinib by targeting heme oxygenase-1
}

\author{
C. GAO, F. H. PENG, L. K. PENG* \\ Department of Urological Organ Transplantation, The Second Xiangya Hospital of Central South University, 139 Renmin Road, Changsha, \\ Hunan, P.R. China, 410011
}

${ }^{*}$ Correspondence: gylchygc@126.com

Received January 13, 2014 / Accepted March 31, 2014

\begin{abstract}
Clear-cell renal cell carcinoma is a highly treatment-resistant tumor type. Heme oxygenase-1 plays an anti-apoptotic role in cancer chemotherapeutic inducing tumor-progression. The miR-200 family was involved in the process of mesenchymal-epithelial transition (MET) during renal development. In the present study, we demonstrated the regulatory relationship between miR-200c and HO-1. We provided evidences to elucidate that miR-200c could sensitize ccRCC cells to sorafenib or imatinib to inhibit cell proliferation, at least partly by targeting HO-1. Moreover, the correlation between miR-200c and HO-1 expression level and drug resistance in ccRCC was also determined. Combined application with chemotherapeutic drugs, miR-200c, a HO-1 inhibitor, may enhance the efficiency of therapy by promoting both apoptosis and autophagy.
\end{abstract}

Key words: clear-cell renal cell carcinoma, resistance, miR-200c, heme oxygenase-1, autophagy

Renal cell carcinoma (RCC), one of the leading lethal causes of cancers, affects about 120,000 deaths worldwide annually with steadily rising. The large part $(\sim 75 \%)$ of cases is clear-cell RCC (ccRCC), which is a highly treatment-resistant tumor type. Emerging investigates are elucidating the molecular mechanisms underlying RCC and promising targets for therapeutics. In clear-cell RCC, mutations of genes result in the up-regulation or down-regulation of many proteins necessary for tumor growth, survival and angiogenesis, such as vascular endothelial growth factor (VEGF), and platelet-derived growth factor (PDGF)[1]. Sorafinib (Nexavar, BAY 43-9006), one of the potent inhibitors of the class of Raf kinase, has been applied in clinical. Sorafinib is an orally available, novel bi-aryl urea compound that represses tumor growth and metastases by targeting the receptor tyrosine kinases VEGFR and PDGFR to inhibit angiogenesis and proliferation[2]. Imatinib (Gleevec), an inhibitor of bcr-abl, PDGF receptor (PDGFR) and the c-kit tyrosine kinases, has been approved by the Food and Drug Administration (FDA) for the treatment of gastrointestinal stromal tumors[3].

Heme oxygenase-1 (HO1), the rate-limiting enzyme in heme degradation with a strong anti-inflammatory and antiapoptotic role, can protect against various injuries, such as ischemic-reperfusion injury[4]. However, the advantages in anti-apoptotic and cytoprotective roles of HO-1 turn out to induce tumor-progression in cancer chemotherapeutic[5]. It was reported that increased expression of $\mathrm{HO}-1$ in human malignant cancer tissue was observed, such as lung cancers[6]. One of reports showed that high expression of HO-1 was associated with poor prognosis in lung cancer[7]. On the contrary, other studies reported that high HO-1 expression was also associated with favorable prognosis in colorectal cancer patients. Moreover, it was recently reported that HO1 nuclear localization was involved in imatinib resistance in chronic myeloid leukemia cells[8], indicating the increased significance of HO-1 in cancer biology. Despite of these conflicting results, it is necessary to determine the role of $\mathrm{HO}-1$ in human malignant tumors.

MicroRNAs(miRNAs), about 22 nt long noncoding regulatory RNAs, show promise as diagnostic biomarkers and therapeutic targets for cancer [9]. Aberrant expression of miRNAs, as oncogenes or tumor suppressors, plays an important role in the pathogenesis, invasion and metastasis of various malignant tumors. Deregulation of several miRNAs has been found in nephroblastomas. Most of the downregulated miRNAs were found silenced through epigenetic 
mechanisms in solid cancers including breast cancer[10] and neuroblastoma[11]. The tissues of ovarian tumors and nephroblastomas showed a significantly decreased expression of miR-185[12]. The miR-200 family was involved in the process of mesenchymal-epithelial transition (MET) during renal development[13]. A recent study found that miR-200c was dramatically decreased in clear cell renal carcinomas compared with non-malignant samples[14]. It was showed that restoration of mature miR-200c in renal cell carcinoma markedly inhibited cancer cell proliferation, suggesting that miR-200c might be a candidate tumor suppressor. However, the precise mechanisms underlying regulation of miR-200c in ccRCC remain unclear.

In this study, we aim to investigate the relationship between HO- 1 and miR-200c in chemotherapeutic resistance in ccRCC, and explore their functions involved with apoptosis and autophagy. In the present study, we demonstrated the regulatory relationship between miR-200c and HO-1. We provided evidences to elucidate that miR-200c, at least partly by targeting $\mathrm{HO}-1$, could sensitize ccRCC cells to sorafenib or imatinib to inhibit cell proliferation via apoptosis and autophagy pathway.

\section{Materials and methods}

Cells culture and treatment. HEK293, SN12C, ACHN, 786-O and Caki- 1 cells, were obtained from American type culture collection (ATCC) and cultivated in RPMI-1640 medium with fetal bovine serum to a final concentration of $10 \%$. All cells cultured in the conditions: $95 \%$ air and 5\% carbon dioxide $(\mathrm{CO} 2)$ at $37^{\circ} \mathrm{C}$.

Sorafenib- or imatinib-resistant 786-O and Caki- 1 cells were treated with gradually increasing concentrations of sorafenib $(1 \mu \mathrm{M}$ to $20 \mu \mathrm{M})$ or imatinib $(1 \mathrm{mmol} / \mathrm{L}$ to $20 \mathrm{mmol} / \mathrm{L}$ ) in cell culture medium. These cells were used as resistant RCC cell lines for subsequent experiments. Ectopic expression of miR-200c in cells was achieved by transfection with pre-miR-200c or anti-miR-200c using Lipofectamine2000 (Invitrogen, USA). Knockdown of HO-1 was performed using HO-1 siRNA (Qiagen). Cells were plated in 24-well plates and transfected for 24 hours or 48 hours. Total RNA or protein was extracted from the indicated cells for analysis.

Reagents. Rabbit polyclonal antibody of $\mathrm{HO}-1$ was purchased from Assay Biotech (Assay Biotech, USA). Mouse polyclonal antibodies of Beclin-1, LC3B, Bcl-xl, Bcl-2, Bid, Bax, and mouse monoclonal antibody of $\beta$-actin were purchased from ImmunoWay (ImmunoWay, USA). Sorafenib and imatinib were obtained from Sigma (Sigma, USA).

Quantitative PCR (qPCR) assay. Qiagen RNeasy Kit was used to extract total RNA of the indicated cells, according to the manufacturer's instructions. Expression of HO-1 mRNA was detected by SYBR green qPCR assay (BioRad, USA). Expression of $\beta$-actin was used as an endogenous control. The specific primers are as follow: HO-1, F: AGGCAGAG-
GGTGATAGAAGAGGC, R: TGAGTGTAAGGACCCA TCGGAGA; $\beta$-actin, F: AGGGGCCGGACTCGTCATACT, R: GGCGGCACCACCATGTAC CCT. MiScript Reverse Transcription kit was used to reverse transcribe RNA into cDNA and MiScript SYBR-Green PCR Kit was used for real time PCR to detect the expression of miRNAs. The specific primers sets for miRNAs and U6 were purchased from GeneCopoeia. Expression of U6 was used as an endogenous control. Data were analyzed through $2^{-\Delta \Delta C T}$ method.

Methylation specific PCR (MSP). Genomic DNA was extracted using the TaKaRa Genomic DNA Extraction Kit (TaKaRa Co., China). Genomic DNA (1 $\mu$ g per sample) was modified with bisulfite using the Epitect Bisulfite Kit Protocol (Qiagen) followed the manufacturer's instructions. Methylation-specific PCR (MS-PCR) was performed on bisulfate-treated DNA. The primers used were miR-200c-UMSP-F: GAATTTGGGGTTTTAAAGTTTTTTT, miR-200c-UMSP-R: CACACCCTAAATCACTAATCACAAA; and miR-200c-MSPF: GAATTTGGGGTTTTAAAGTTTTTTC

miR-200c-MSP-R: CACCCTAAATCGCTAATCACG. The annealing temperature was $60^{\circ} \mathrm{C}$ for Methylated -PCR, and $55^{\circ} \mathrm{C}$ for Un-methylated -PCR, with 27 cycles used for each. The size of MS-PCR products were $276 \mathrm{bp}$ and $278 \mathrm{bp}$ for miR200c-MSP and miR-200c-UMSP, respectively.

Western blotting. Total protein $(60 \mu \mathrm{g})$ extracted from indicated cells was separated on SDS-polyacrylamide gels for HO-1, Beclin-1, Bcl-xl, Bim, Bax and $\beta$-actin detection. $\beta$-actin was used as loading control. The protein in gels was transferred to nitrocellulose membranes, and then incubated with the indicated antibodies in recommended dilution for overnight at $4^{\circ} \mathrm{C}$. Then membranes were washed with $0.1 \mathrm{M}$ PBST and incubated with HRP-conjugated secondary antibody. Signals were visualized using ECL Substrates and quantified using Optiquant software.

CCK-8 cell proliferation assay. Cell Counting Kit-8 (CCK8) (Solarbio, China) was used to evaluate cell proliferation rates. $0.5 \times 10^{4}$ cells were seeded in each 96 -well plate for $24 \mathrm{~h}$, transfected with the indicated miRNA or siRNA, and further incubated for $0 \mathrm{~h}, 24 \mathrm{~h}$, and $48 \mathrm{~h}$ respectively. $1 \mathrm{~h}$ before ending of incubation, $10 \mu \mathrm{l}$ CCK-8 reagents was added to each well. OD $450 \mathrm{~nm}$ value in each well was determined by an enzyme immunoassay analyzer.

Dual luciferase reporter assay. The indicated cells were seeded in 96-well plates the day before transfection. Then, cells were cotransfected with $25 \mathrm{ng}$ pmiR-report 3'UTR HO-1 target gene and $10 \mathrm{ng}$ pRL-TK (Renilla plasmid), and $15 \mathrm{nM}$ pre-miR-200c or anti-miR-200c using Lipofectamine 2000. Cells were transfected for 48 hours with Dual-LuciferaseReporter Assay System and luminescence was detected using a luminometer (Promega). The luciferase activity was normalized to the Renilla activity.

Statistical analysis. Data are expressed as mean \pm SD and analyzed by Student's t-test. Compared with respective controls, $P$ values of $<0.05$ were considered statistically significant. 


\section{Result}

Expression levels of miR-200c and HO-1 in RCC cell lines treated with gradually increasing concentrations of sorafenib or imatinib. The expression levels of miR-200c were significantly lower in RCC cell lines (786-O, caki-1) treated with sorafenib or imatinib than control (Figure 1A). Likewise, HO-1 expression was significantly increased at mRNA and protein levels in RCC cell lines (786-O, caki-1) treated with sorafenib or imatinib compared with control group (Figure 1B, C). However, we did not found significant changes in other
miRNAs(miR-200a, miR-200b, miR-429 and miR-141) in our study (Figure 1E).

Methylation in the miR-200c promoter. The expression level of miR-200c was significantly lower in RCC cell lines treated with sorafenib or imatinib than control. In light of this, we suspected the epigenetic mechanisms may be involved in the silencing of miR-200c. The miR-200c methylation status was examined using MSP. As shown in Figure 1F, the promoter region of miR-200c was unmethylated in normal renal cells(HEK293), whereas it was hypermethylated in renal carcinoma cell lines(SN12C, ACHN, 786-O and Caki-1).
A

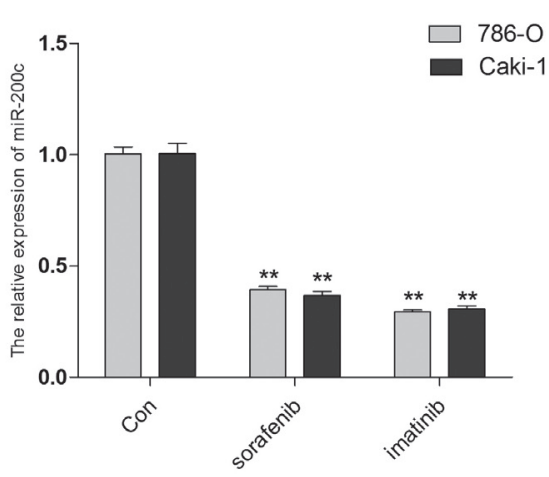

C



E

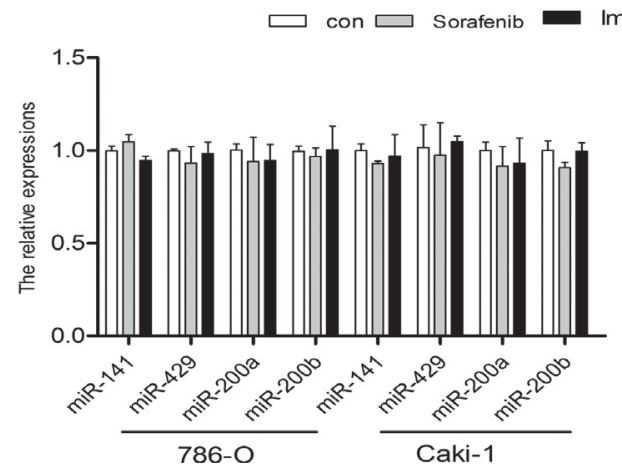

B

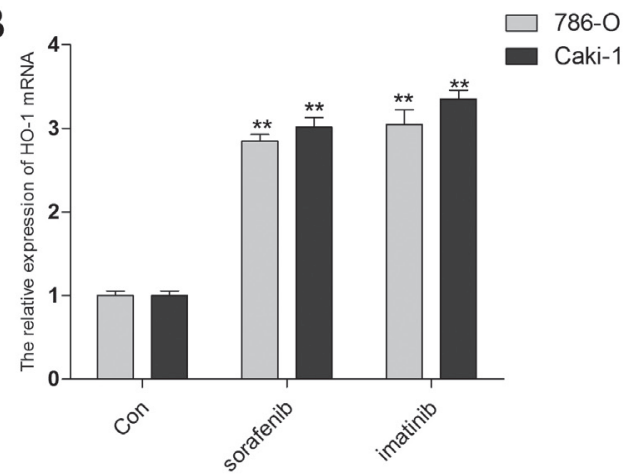

D

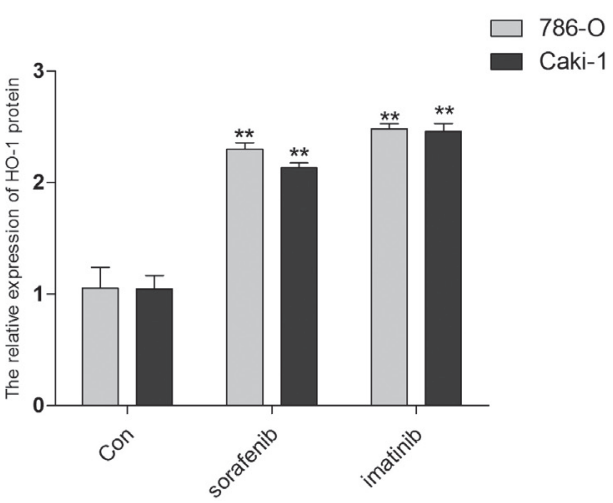

F

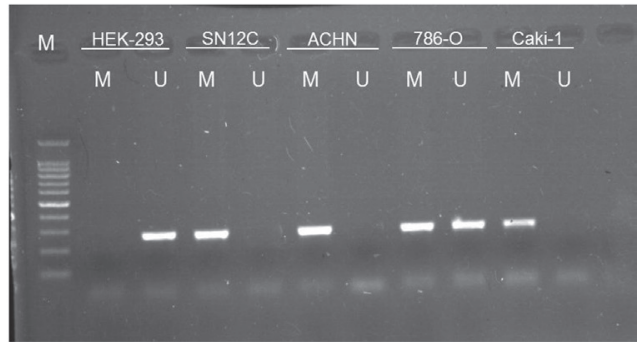

Figure 1. Expression of HO-1 and miR-200c in sorafenib- or imatinib-resistant 786-O and Caki-1 cells. (A) qPCR detected the expression of miR-200c; (B) qPCR detected the expression of HO-1 mRNA.(C, D) western blot the expression of HO-1 protein and quantification. (E) qPCR detected the expression of miR-200a, miR-200b, miR-141 and miR-492. (F) MSP examined the miR-200c promoter methylation status. M, Methylation; U, Unmethylation. $\left({ }^{\star *} \mathrm{p}<0.01\right.$ versus control group. Data shown are means \pm S.D. $)$ 
A

3'UTR of HO-1

$\begin{array}{llll}\text { wt-HO-1 } & 5^{\prime} & \text {... UCAGCAUCCUCAGUU CCUGCAGC... } & 3^{\prime}\end{array}$

miR-200c 3" GAGUUUCGUUUG G GACGUCU

mut-HO-1 5' ... UCAGCAUCCUCAGUUG GACG U CC... $3^{\prime}$

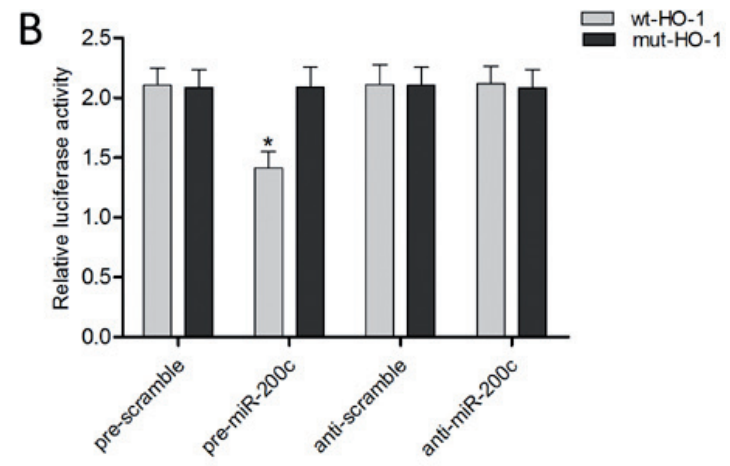

$\mathrm{D}$



$\mathrm{F}$

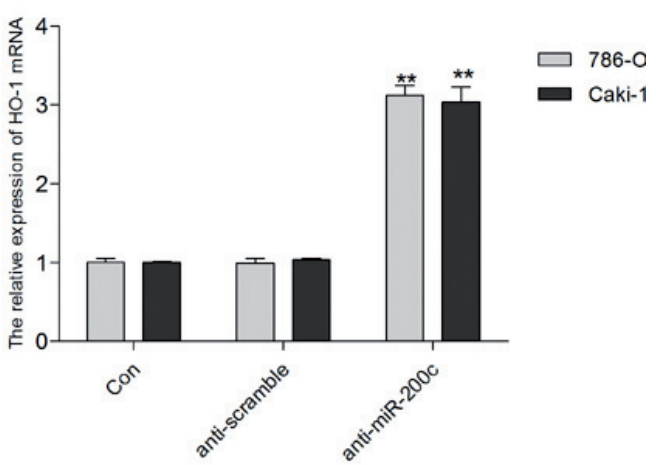

G

$E$

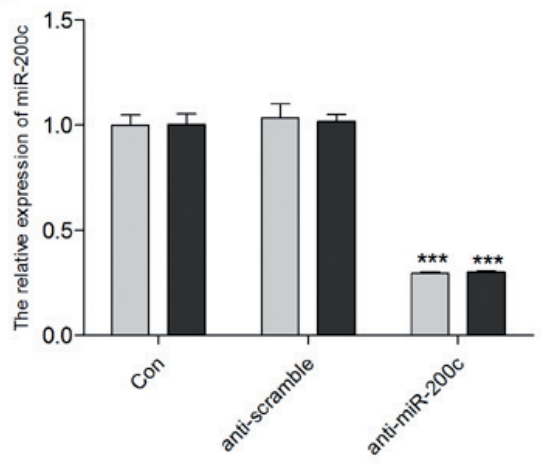

786-0

Caki-1

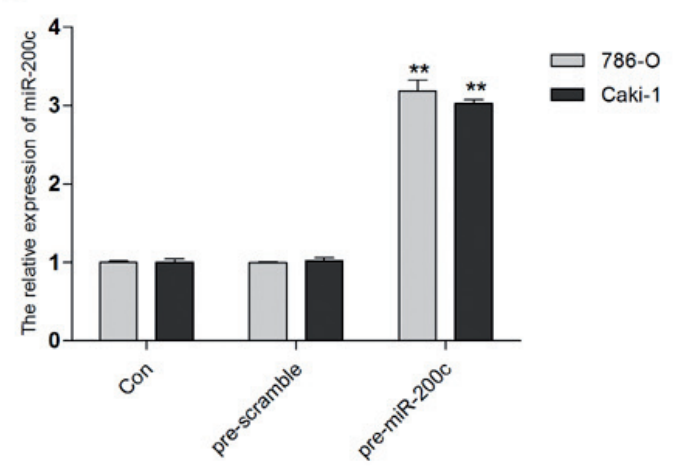

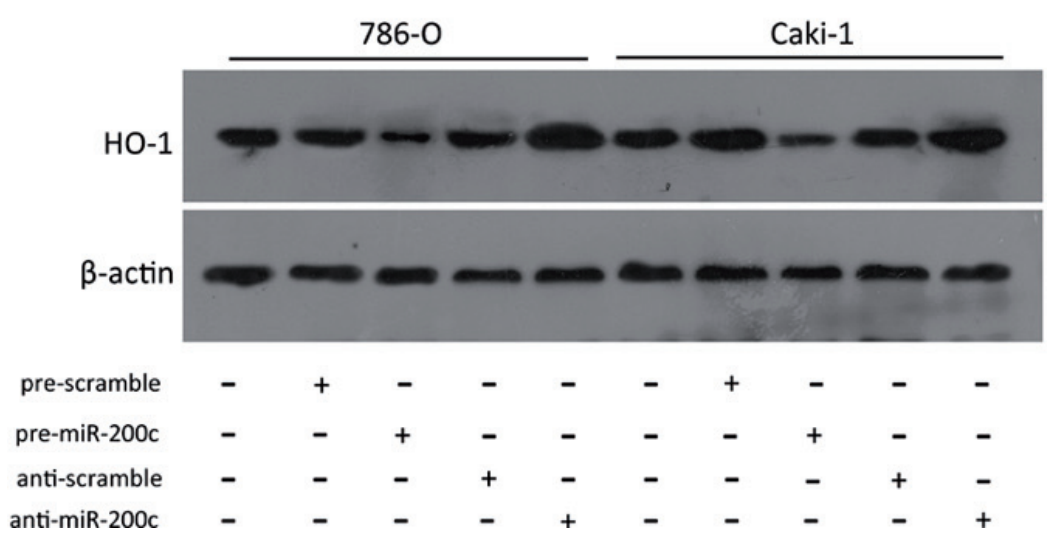

Figure 2. miR-200c directly targets HO-1. (A) The predicted miR-200c binding site within HO-1 3'UTR and its mutated version. (B) The repression of luciferase activity by HO-1 3'UTR was dependent on miR-200c. Mutated HO-1 3'UTR abrogated miR-200c mediated repression luciferase activity. (C) qPCR detected the expression of miR-200c after pre-miR-200c transfection in 786-O and Caki-1 cells. (D) qPCR detected the expression of HO-1 mRNA after pre-miR-200c transfection in 786-O and Caki-1 cells. (E) qPCR detected the expression of miR-200c after anti-miR-200c transfection in 786-O and Caki-1 cells. (F) qPCR detected the expression of HO-1 mRNA after anti-miR-200c transfection in 786-O and Caki- 1 cells. (G) HO-1 protein expression in 786-O and Caki-1 cells treated with pre-miR-200c or anti-miR-200c was obtained by Western blot. $\beta$-actin was used as the loading control. $\left({ }^{*} P<0.05,{ }^{* *} P<0.01,{ }^{* * *} P<0.001\right.$ versus control, data shown are means \pm S.D.) 

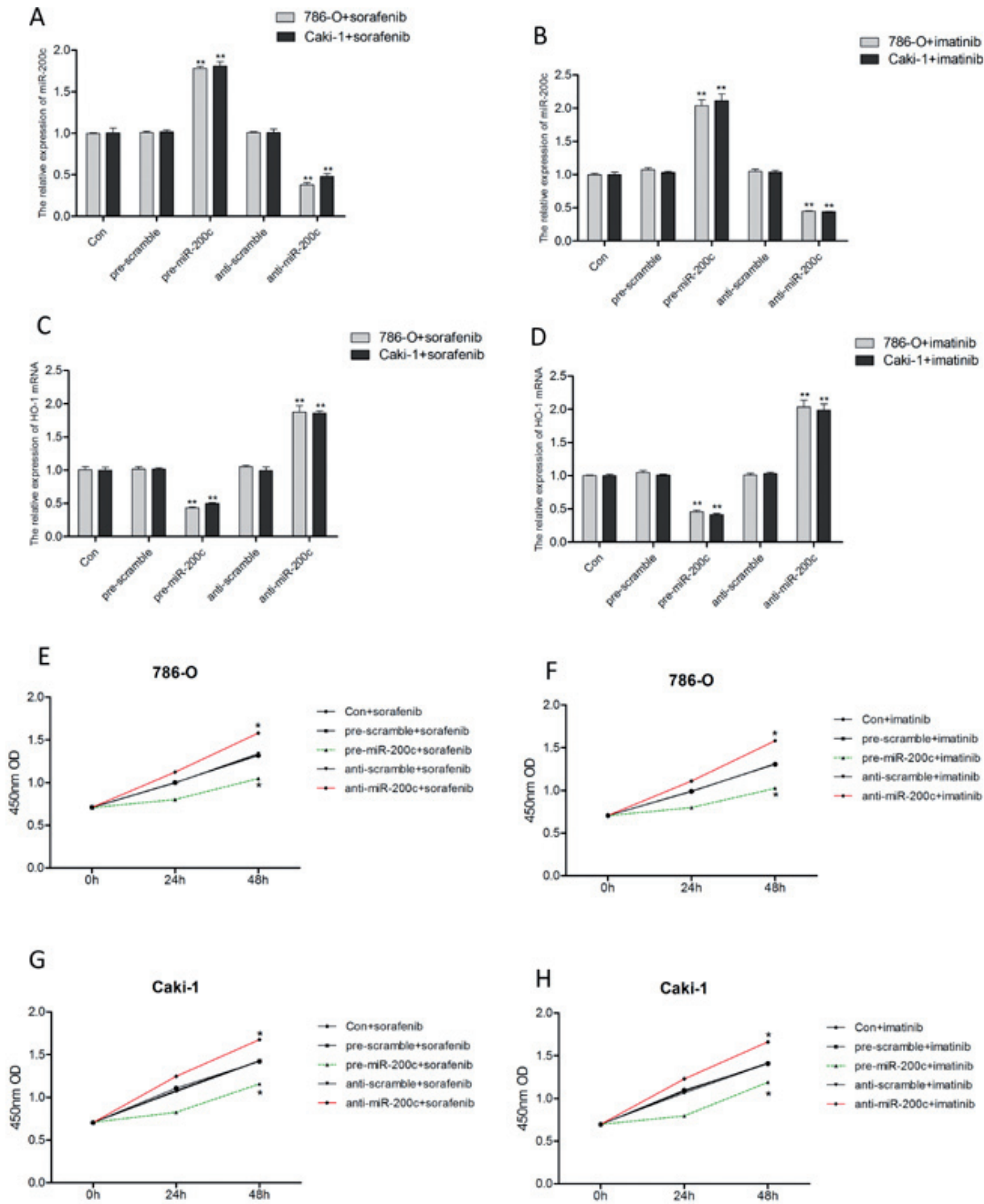

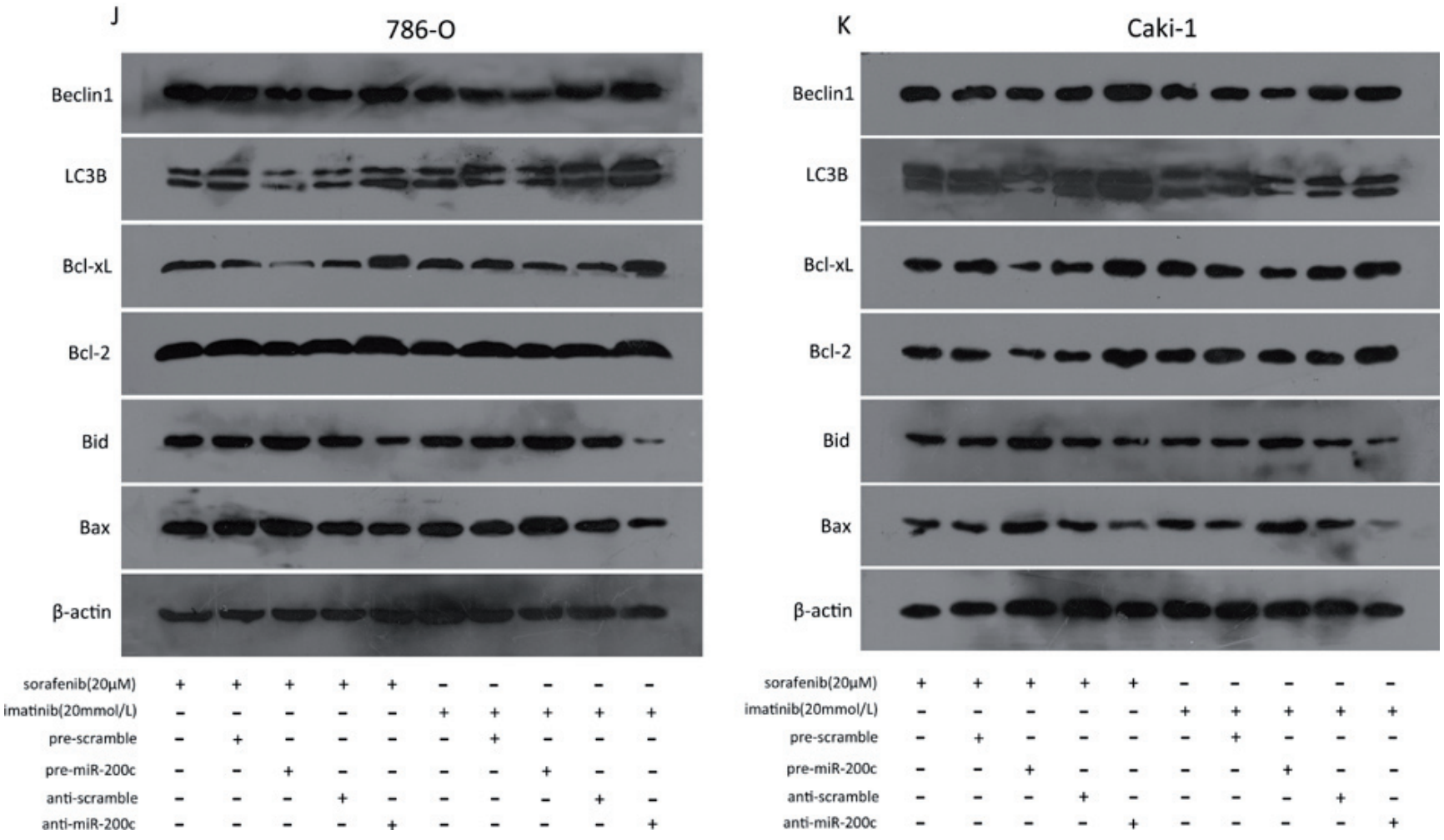

Figure 3. Synergistic effect of miR-200c with sorafenib or imatinib on RCC cells proliferation, and regulated the expression of Beclin1, LC3B, Bcl-xl, Bcl-2, Bid and bax in 786-O and Caki-1 cells. (A, B) qPCR detected the expression of miR-200c after sorafenib or imatinib treatment in 786-O and Caki1 cells transfected with pre-miR-200c or anti-miR-200c. (C, D) qPCR detected the expression of HO-1 after sorafenib or imatinib treatment in 786-O and Caki-1 cells transfected with pre-miR-200c or anti-miR-200c. (E, F, G, H) Cell growth was evaluated by CCK8 assay. The data were presented as 450 nm optical density. (J, K)Western blotting detected the expression of Beclin1, LC3B, Bcl-xl, Bcl-2, Bid and bax after sorafenib or imatinib treatment in 786-O and Caki-1 cells transfected with pre-miR-200c or anti-miR-200c. $\left({ }^{\star} P<0.05,{ }^{*} P<0.01\right.$ versus control, data shown are means \pm S.D. $)$

miR-200c directly targets HO-1 in RCC cell lines. Ectopic expression of miR-200c in cells was achieved efficiently by transfection with pre-miR-200c or anti-miR-200c using Lipofectamine2000 (Figure 2C, E). Quantitative real-time PCR assays showed that mRNA expression levels of HO- 1 in the 786-O and caki- 1 cell lines transfected with pre-miR-200c were lower than those of transfected with pre-scramble (Figure 2D), vice versa, anti-miR-200c transfection upregulated the expression of HO-1 mRNA in 786-O and caki-1 cell lines (Figure 2F). Furthermore, HO-1 protein expression levels were markedly downregulated by pre-miR-200c transfection, and were upregulated by anti-miR-200c transfection, compared with the control in 786-O and caki-1cell lines (Figure 2G).

To identify whether the 3'-untranscriptional region (3'UTR) of HO-1 had a direct target site for miR-200c or not, a dual luciferase reporter assay was performed using a vector encoding the wild type (Wt) and mutant (Mut) 3'UTR of HO-1 mRNA (Figure 2A), and it was found that the luminescence activity was significantly repressed in the miR-200C transfectants compared to the control-transfectant (Figure 2B). Moreover, miR-200c-mediated repression of luciferase activity was abolished by the mutant type 3'UTR of HO-1 (Figure 2B). These results determine that miR-200c directly targets $\mathrm{HO}-1$ and regulates its expression at transcriptional level. (Figure 2D)

Synergistic effect of miR-200c with sorafenib or imatinib on RCC cells proliferation, and regulated the expression of Beclin1, LC3B, Bcl-xl, Bcl-2, Bid and bax in 786-O and Caki1 cells. To validate synergistic effect of miR-200c with sorafenib or imatinib in RCC cell growth, CCK assay was performed by treated with sorafenib or imatinib after transfecting pre-miR$200 \mathrm{c} \mathrm{mimics}$ or anti-miR-200c into RCC cells (Figure 3A, B, C, $D)$. As the data shown, we observed that sorafenib or imatinib treatment significantly decreased cell proliferation in 786-O and Caki-1 cells transfected with pre-miR-200c (Figure 3E, F, $H, G$ ), compared to the cells treated with sorafenib or imatinib alone and pre-scramble control. Besides, we observed that downregulation of miR-200c by transfecting anti-miR-200c in 786-O and Caki- 1 cells increased significantly cell proliferation, which was inhibited by sorafenib or imatinib treatment (Figure $3 \mathrm{E}, \mathrm{F}, \mathrm{H}, \mathrm{G}$ ). It suggested that there was a synergistic effect between miR-200c and sorafenib or imatinib in inhibiting the proliferation of RCC cells. 
A

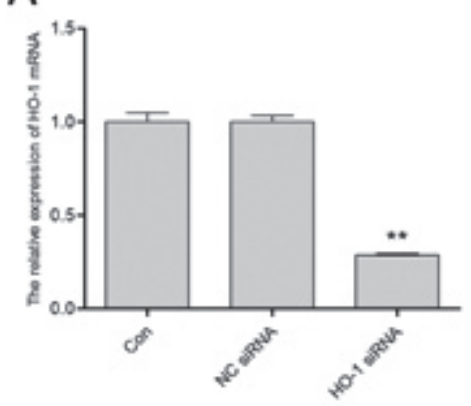

D

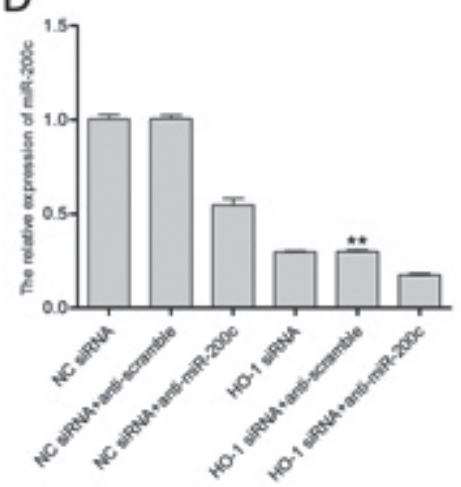

B

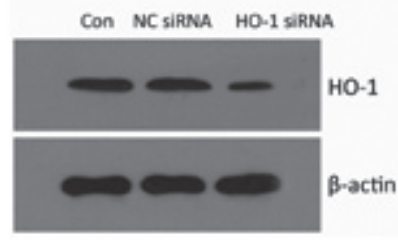

E

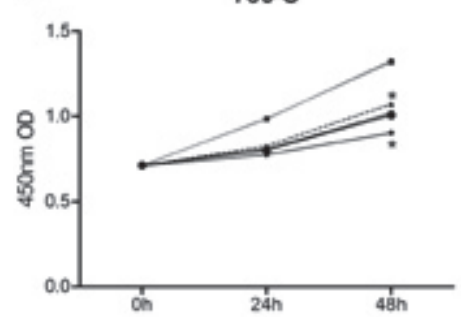

- NC siFana

- NC sifova+pre-scrambie

-2. NC sifova+pre-miR-200C

- HO-1 siRva

- HO-1 siR2A+pre-miR-2000

- HO-1 siReAtpre-scramble
C

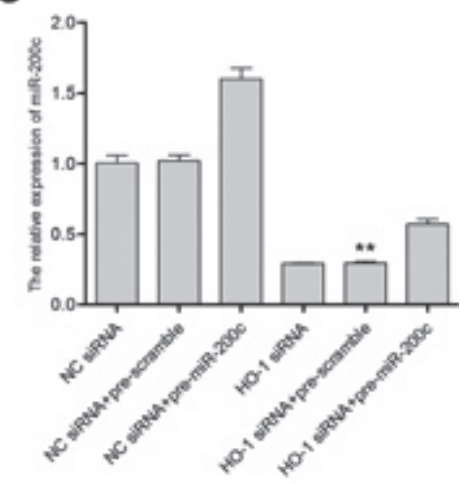

F

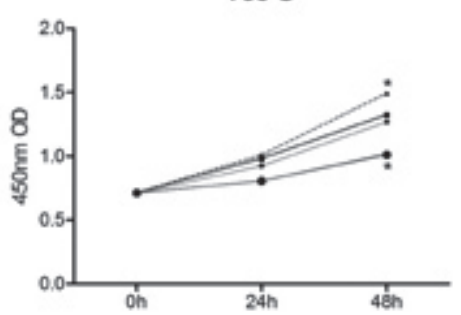

- NC sirna

- NC siRNA+anti-scramble

- - NC sipNAtanti-miR-200c

- ho-1 sirva

- HO-1 siRNA+anti-miR-200C

- HO-1 siRNA+anti-scramble
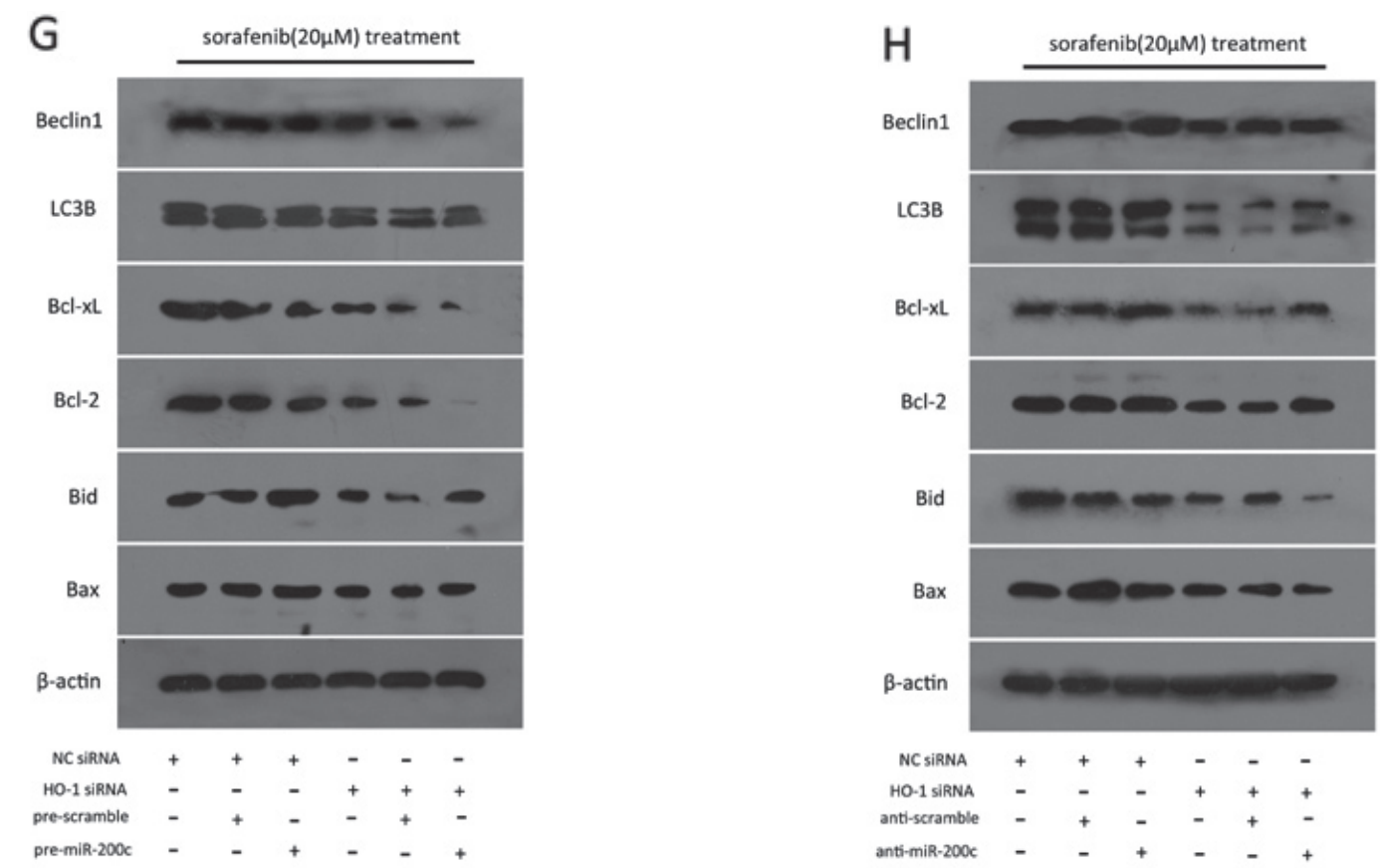

Figure 4. Knockdown of HO-1 expression repress RCC cell proliferation, and regulated the expression of Beclin1, LC3B, Bcl-xl, Bcl-2, Bid and bax in 786-O cells. (A, B) qPCR and western blot detected the expression of HO-1 after sorafenib treatment in 786-O transfected with HO- 1 siRNA. (C, D) QPCR detected the expression of miR-200c after sorafenib treatment in 786-O co-transfected with HO-1 siRNA and pre-miR-200c or antimiR-200c. (E, F) Cell growth was evaluated by CCK8 assay. The data were presented as $450 \mathrm{~nm}$ optical density. (G, H)Western blotting detected the expression of Beclin1, LC3B, Bcl-xl, Bcl-2, Bid and bax after sorafenib treatment in 786-O co-transfected with HO-1 siRNA and pre-miR-200c or anti-miR-200c. $\left({ }^{\star} P<0.05,{ }^{\star *} P<0.01\right.$ versus control, data shown are means \pm S.D. $)$ 
To explore potential molecular mechanisms underlying miR-200c-induced cell apoptosis and autophagy, we tested the expression of apoptosis- and autophagy-related genes including autophagy-related Beclin1 and LC3B, anti-apoptosis gene $\mathrm{Bcl}-\mathrm{xl}$ and $\mathrm{Bcl} 2$, pro-apoptosis gene Bid and bax. Overexpression of miR-200c downregulated significantly the expression of Beclin1, LC3B, Bcl-xl and Bcl2 proteins, and upregulated dramatically the expression of Bid and bax proteins compared to that in the control cells treated with sorafenib or imatinib alone. However, when knockdown the expression of miR-200c, upregulation of Beclin1, LC3B, Bcl-xl and $\mathrm{Bcl} 2$ proteins and downregulation of Bid and bax proteins were also observed (Figure 3J, K).

Knockdown of HO-1 expression repress RCC cell proliferation, and regulated the expression of Beclin1, LC3B, Bcl-xl, Bcl-2, Bid and bax in 786-O. To further confirm the potential relationship between miR-200c and the downstream gene $\mathrm{HO}-1$, we tested cell proliferation under the condition of siRNA mediated knockdown of HO-1 gene. Once HO-1 expression was effectively decreased by siRNA (Figure 4A, B, C, D), transfected 786-O cells exhibited inhibited cell proliferation (Figure 4E, F), which was in consistent with the inhibitory effects induced by upregulation of miR-200c. Furthermore, when treating 786-O cells with HO-1 siRNA in combination with miR-200c mimics, we observed synergistic inhibitory effects on cell proliferation (Figure 4E, F), compared to either HO-1 siRNA or miR-200c mimics treatment alone.

To demonstrate relationship between HO-1, a target of miR-200c, and cell apoptosis and autophagy, we tested the expression of apoptosis- and autophagy-related genes including autophagy-related Beclin1 and LC3B, anti-apoptosis gene $\mathrm{Bcl}-\mathrm{xl}$ and $\mathrm{Bcl} 2$, pro-apoptosis gene Bid and bax. Downregulation of HO-1 downregulated significantly the expression of Beclin1, LC3B, Bcl-xl and Bcl2 proteins, and upregulated dramatically the expression of Bid and bax proteins compared to that in the control cells treated with sorafenib alone. Furthermore, when treating 786-O cells with HO-1 siRNA in combination with miR-200c mimics, synergistic effects on decreasing apoptosis- and autophagyrelated genes were observed, compared to either HO-1 siRNA or miR-200c mimics treatment alone. Whereas combining HO-1 siRNA with anti-miR-200c reversed the effects of HO-1 siRNA treatment. (Figure 4G, H)

\section{Discussion}

Downregulation of miR-200c was observed in the whole RCC succession. It was reported that miR-200c could suppress epithelial-to-mesenchymal transition (EMT)-driven resistance, regulate the sensitivity of chemotherapy in gastric cancer[15], and contribute to antiestrogen resistance in human breast cancer [16]. Based on these findings, we speculated miR$200 \mathrm{c}$ might be also involved in ccRCC treatment-resistant. In our recent research, we confirmed the relationship between
miR-200c and HO-1, and found that miR-200c inhibited ccRCC cell proliferation by targeting HO- 1 .

By quantitative PCR, we validated that miR-200c was dramatically downregulated in ccRCC cell lines treatmentresistant to sorafenib or imatinib, which were consistent with previous studies on other cancers. Epigenetic modifications were prevalent in many cancers, and many research groups have reported comparisons of alterations in methylated microRNA expression for cancers[17]. MiRNAs themselves were able to be regulated by epigenetic mechanisms[18]. Here, we found that the promoter region of miR-200c was hypermethylated in renal carcinoma cell lines treated with sorafenib or imatinib than control, suggesting epigenetic mechanisms might be also involved in ccRCC treatment-resistant. We restored miR-200c expression before sorafenib or imatinib treatment in 786-O and Caki- 1 cells and found that miR-200c repressed cell proliferation. On the contrary, when transfected with anti-miR-200c to decrease the expression of miR-200c in 786-O and Caki-1 cells, the cells exhibited stimulated proliferation capabilities. These results suggested that miR-200c was involved in the ccRCC treatment-resistant.

Heme oxygenase-1 (HO1), an anti-apoptotic gene, was involved in imatinib resistance in cancers[19] and the regulation of autophagy[20]. Mechanically, HO-1 transcriptionally involved in Beclin-1 and Bcl-2 signaling pathway, which has been reported essential for tumor growth and autophagy[21]. Our findings demonstrated a vital molecular relationship between miR-200c and HO-1. Our results showed that upregulation of miR-200c expression in 786-O and Caki- 1 cells effectively repressed HO-1 expression at both mRNA and protein level, whereas downregulation of miR-200c dramatically promoted HO-1 expression in the two cell lines. It indicated a potential inverse relevance of $\mathrm{miR}-200 \mathrm{c}$ and $\mathrm{HO}-1$ in ccRCC. Furthermore, by luciferase reporter assay, it was verified that miR-200c directly targeted HO-1 gene by binding to specific complementary site within its 3' UTR. Through gain-of-function study by transfecting pre-miR-200c before sorafenib or imatinib treatment in 786-O and Caki- 1 cells, we found that upregulation of miR-200c contributed to promote sensitivity of drug treatment on cellular proliferation, which was also confirmed in HO-1 loss-of-function study by siRNA mediated knockdown. Additionally, the inverse relationship of expression between miR-200c and HO-1 expression in ccRCC cell lines indicated that downregulation of miR-200c may account for upregulation of HO- 1 in ccRCC drug treatment resistance. Taken together, these findings consolidated our hypothesis that upregulation of miR-200c sensitized 786-O and Caki- 1 cells to sorafenib or imatinib treatment, at least partially, by directly targeting HO-1 to inhibit cellular proliferation.

The two parts of pro- (Bax, Bak, Bad) and anti-apoptotic (Bcl-2, Bcl-xL) members comprised Bcl2 family, which played as a key regulator of apoptosis [22] and a crucial factor in tumor development. Thus, upregulation of anti-apoptotic members, or downregulation of the pro-apoptotic members of the Bcl-2 family would result in tumor progression. It has 
been found that increased expression of $\mathrm{Bcl}-\mathrm{xL}$ was involved in resistance to chemotherapeutic drugs [23]. In our recent study, we found that decreased expression of $\mathrm{HO}-1$ induced by pre-miR-200c or siRNA-HO-1 transfection markedly reduced Bcl-xL, and increased Bid and Bax in renal cancer cells, with slight change but not significant in expression of Bcl-2. Although both Bcl-xL and Bcl-2 are known to enhance antiapoptotic signals, it is considered that $\mathrm{Bcl}-\mathrm{xL}$ was involved in chemoresistance. According previous studies and our recent results, it is possible that promotion of apoptosis by modulation of $\mathrm{Bcl}-\mathrm{xL}, \mathrm{Bid}$ and $\mathrm{Bax}$ is one of the possible mechanisms underlying miR-200c-mediated cancer cell death by targeting HO-1. Furthermore, recent studies indicate that cancer cell death is also controlled by autophagy interplay with apoptosis. During late or advanced stages of cancer, by supplementing the growing needs of the cells with excess supply of oxygen and nutrients, autophagy comes as a savior in cancer[24]. Moreover, some reports showed that autophagy promoted apoptotic functions of chemotherapeutic drugs, but others indicated that it may act as a survival factor of cancer cells in drugs treatment [25]. Our recent results showed that upregulation of miR-200c or downregulation of HO-1 decreased some key mediators (Beclin-1 and LC3B) of the autophagy pathway. Beclin-1, one of the most critical molecules in the autophagy pathway, is an integral part of PI3K multiprotein complex, which was essential for autophagosome nucleation. The interaction between Beclin-1 and anti-apoptotic members of $\mathrm{Bcl}-2$ family has been demonstrated. Zalckvar and his colleagues reported that the dissociation of Beclin-1 from anti-apoptotic members of $\mathrm{Bcl}-2$ family promotes autophagy[26]. Our findings indicated that miR-200c inhibited the association of Beclin-1 with its inhibitor Bcl-xL promoted by HO-1, and thereby may reduce autophagy. Of note, instead of protecting cells from apoptosis, autophagy can mediate cell death under specific circumstances[27]. It was reported that chemotherapeutic drugs promoted autophagy to kill tumor cells[28], suggesting that cells may not only die by apoptosis, but also autophagy. We demonstrate that inhibition of both apoptosis and autophagy play a key role in miR-200c-mediated death of renal cancer cells.

In conclusion, this study found newly an important mechanism underlying miR-200c-mediated chemotherapeutic resistance of renal cancer cells. MiR-200c promoted cells to death by targeting to HO-1 to suppress both apoptosis and autophagy induced by sorafenib or imatinib. Thus, our work highlighted the significance of miR-200c as a potential therapeutic target for clear-cell renal cell carcinoma. Combined application with chemotherapeutic drugs, overexpression of miR-200c may enhance the efficiency of therapy.

\section{References}

[1] OHH M, PARK CW, IVAN M, HOFFMAN MA, KIM TY, et al. Ubiquitination of hypoxia-inducible factor requires direct binding to the beta-domain of the von Hippel-Lindau protein.
Nat Cell Biol, 2000, 2: 423-427 http://dx.doi.org/10.1038/ 35017054

[2] WILHELM SM, CARTER C, TANG L, WILKIE D, MCNABOLA A, et al. BAY 43-9006 exhibits broad spectrum oral antitumor activity and targets the RAF/MEK/ERK pathway and receptor tyrosine kinases involved in tumor progression and angiogenesis. Cancer Res, 2004, 64: 7099-7109 http:// dx.doi.org/10.1158/0008-5472.CAN-04-1443

[3] DEMETRI GD, VON MEHREN M, BLANKE CD, VAN DEN ABBEELE AD, EISENBERG B, et al. Efficacy and safety of imatinib mesylate in advanced gastrointestinal stromal tumors. N Engl J Med, 2002, 347: 472-480 http://dx.doi. org/10.1056/NEJMoa020461

[4] RICHARDS JA, WIGMORE SJ, DEVEY LR. Heme oxygenase system in hepatic ischemia-reperfusion injury. World J Gastroenterol, 2010, 16: 6068-6078 http://dx.doi.org/10.3748/wjg. v16.i48.6068

[5] BANERJEE P, BASU A, WEGIEL B, OTTERBEIN LE, MIZUMURA K, et al. Heme oxygenase-1 promotes survival of renal cancer cells through modulation of apoptosis- and autophagy-regulating molecules. J Biol Chem, 2012, 287: 32113-32123 http://dx.doi.org/10.1074/jbc.M112.393140

[6] DEGESE MS, MENDIZABAL JE, GANDINI NA, GUTKIND JS, MOLINOLO A, et al. Expression of heme oxygenase-1 in non-small cell lung cancer (NSCLC) and its correlation with clinical data. Lung Cancer, 2012, 77: 168-175 http://dx.doi. org/10.1016/j.lungcan.2012.02.016

[7] TSAI JR, WANG HM, LIU PL, CHEN YH, YANG MC, et al. High expression of heme oxygenase- 1 is associated with tumor invasiveness and poor clinical outcome in non-small cell lung cancer patients. Cell Oncol (Dordr), 2012, 35: 461-471 http://dx.doi.org/10.1007/s13402-012-0105-5

[8] TIBULLO D, BARBAGALLO I, GIALLONGO C, LA CAVA P, PARRINELLO N, et al. Nuclear translocation of heme oxygenase-1 confers resistance to imatinib in chronic myeloid leukemia cells. Curr Pharm Des, 2013, 19: 2765-2770 http:// dx.doi.org/10.2174/1381612811319150012

[9] BISHOP JA, BENJAMIN H, CHOLAKH H, CHAJUT A, CLARK DP, et al. Accurate classification of non-small cell lung carcinoma using a novel microRNA-based approach. Clin Cancer Res, 2010, 16: 610-619 http://dx.doi.org/10.1158/ 1078-0432.CCR-09-2638

[10] PNG KJ, YOSHIDA M, ZHANG XH, SHU W, LEE H, et al. MicroRNA-335 inhibits tumor reinitiation and is silenced through genetic and epigenetic mechanisms in human breast cancer. Genes Dev, 2011, 25: 226-231 http://dx.doi. org/10.1101/gad.1974211

[11] DAS S, BRYAN K, BUCKLEY PG, PISKAREVA O, BRAY IM, et al. Modulation of neuroblastoma disease pathogenesis by an extensive network of epigenetically regulated microRNAs. Oncogene, 2013, 32: 2927-2936 http://dx.doi.org/10.1038/ onc.2012.311

[12] IMAM JS, BUDDAVARAPU K, LEE-CHANG JS, GANAPATHY S, CAMOSY C, et al. MicroRNA-185 suppresses tumor growth and progression by targeting the Sixl oncogene in human cancers. Oncogene, 2010, 29: 4971-4979 http://dx.doi. org/10.1038/onc. 2010.233 
[13] WESSELY O, AGRAWAL R, TRAN U. MicroRNAs in kidney development: lessons from the frog. RNA Biol, 2010, 7: 296-299 http://dx.doi.org/10.4161/rna.7.3.11692

[14] SENANAYAKE U, DAS S, VESELY P, ALZOUGHBI W, FROHLICH LF, et al. miR-192, miR-194, miR-215, miR200c and miR-141 are downregulated and their common target ACVR2B is strongly expressed in renal childhood neoplasms. Carcinogenesis, 2012, 33: 1014-1021 http://dx.doi. org/10.1093/carcin/bgs126

[15] CHANG L, GUO F, WANG Y, LV Y, HUO B, et al. MicroRNA200c Regulates the Sensitivity of Chemotherapy of Gastric Cancer SGC7901/DDP Cells by Directly Targeting RhoE. Pathol Oncol Res, 2013,

[16] MANAVALAN TT, TENG Y, LITCHFIELD LM, MULUHNGWI P, AL-RAYYAN N, et al. Reduced expression of miR-200 family members contributes to antiestrogen resistance in LY2 human breast cancer cells. PLoS One, 2013, 8: e62334 http://dx.doi.org/10.1371/journal.pone.0062334

[17] HOGAN LE, MEYER JA, YANG J, WANG J, WONG N, et al. Integrated genomic analysis of relapsed childhood acute lymphoblastic leukemia reveals therapeutic strategies. Blood, 2011, 118: 5218-5226 http://dx.doi.org/10.1182/blood-201104-345595

[18] KELLY TK, DE CARVALHO DD, JONES PA. Epigenetic modifications as therapeutic targets. Nat Biotechnol, 2010, 28: 1069-1078 http://dx.doi.org/10.1038/nbt.1678

[19] TIBULLO D, BARBAGALLO I, GIALLONGO C, LA CAVA P, PARRINELLO N, et al. Nuclear translocation of heme oxygenase- 1 confers resistance to imatinib in chronic myeloid leukemia cells. Curr Pharm Des, 2013, 19: 2765-2770 http:// dx.doi.org/10.2174/1381612811319150012

[20] LONG JS, RYAN KM. New frontiers in promoting tumour cell death: targeting apoptosis, necroptosis and autophagy. Oncogene, 2012, 31: 5045-5060 http://dx.doi.org/10.1038/onc.2012.7
[21] BALAN M, PAL S. A novel CXCR3-B-induced growth-inhibitory signal in cancer cells is mediated through the regulations of Bach-1 and Nrf2 nuclear translocation. J Biol Chem, 2013,

[22] CORY S, HUANG DC, ADAMS JM. The Bcl-2 family: roles in cell survival and oncogenesis. Oncogene, 2003, 22: 8590-8607 http://dx.doi.org/10.1038/sj.onc. 1207102

[23] AMUNDSON SA, MYERS TG, SCUDIERO D, KITADA S, REED JC, et al. An informatics approach identifying markers of chemosensitivity in human cancer cell lines. Cancer Res, 2000, 60: 6101-6110

[24] AMARAVADI RK, LIPPINCOTT-SCHWARTZ J, YIN XM, WEISS WA, TAKEBE N, et al. Principles and current strategies for targeting autophagy for cancer treatment. Clin Cancer Res, 2011, 17: 654-666 http://dx.doi.org/10.1158/1078-0432. CCR-10-2634

[25] BAREFORD MD, PARK MA, YACOUB A, HAMED HA, TANG Y, et al. Sorafenib enhances pemetrexed cytotoxicity through an autophagy-dependent mechanism in cancer cells. Cancer Res, 2011, 71: 4955-4967 http://dx.doi.org/10.1158/ 0008-5472.CAN-11-0898

[26] ZALCKVAR E, BERISSI H, MIZRACHY L, IDELCHUK Y, KOREN I, et al. DAP-kinase-mediated phosphorylation on the $\mathrm{BH} 3$ domain of beclin 1 promotes dissociation of beclin 1 from Bcl-XL and induction of autophagy. EMBO Rep, 2009, 10: 285-292 http://dx.doi.org/10.1038/embor.2008.246

[27] MAIURI MC, ZALCKVAR E, KIMCHI A, KROEMER G. Self-eating and self-killing: crosstalk between autophagy and apoptosis. Nat Rev Mol Cell Biol, 2007, 8: 741-752 http:// dx.doi.org $/ 10.1038 / \mathrm{nrm} 2239$

[28] KONDO Y, KANZAWA T, SAWAYA R, KONDO S. The role of autophagy in cancer development and response to therapy. Nat Rev Cancer, 2005, 5: 726-734 http://dx.doi.org/10.1038/ $\underline{\operatorname{nrc1692}}$ 